\title{
State Transfer of Two-level Quantum System Feedback Control Based on Online State Estimation
}

\author{
Shuang Cong ${ }^{1}$, Yaru Tang ${ }^{1}$, Kezhi $\mathrm{Li}^{2}$ \\ 1. Department of Automation, University of Science and Technology of China, Hefei 230027, P. R. China \\ E-mail: scong@ustc.edu.cn \\ 2. University College London, London, NW12DA, United Kingdom \\ E-mail: Ken.li@ucl.ac.uk
}

\begin{abstract}
A quantum state feedback control method is proposed in this paper. The state of a two-level open quantum system is estimated online based on the continuous weak measurement and the compressed sensing theory. Based on the state estimated online and the Lyapunov stability theorem, the state feedback control law used to transfer the quantum state is designed. Moreover, three numerical simulation experiments are implemented in the MATLAB environment: the state transfer from eigenstates to eigenstates, superposition states to superposition states, and superposition states to mixed states. The experimental results verify high performance of the proposed feedback control based on the state estimated online.
\end{abstract}

Key Words: Two-level quantum system, Online estimation of quantum state, Quantum Lyapunov control, State transfer.

\section{Introduction}

Different from classic measurements, quantum states cannot be obtained from the results of direct measurements, so efficient quantum state reconstruction is required and becomes an attractive research topic. Quantum state tomography (QST), a method of reconstructing quantum states [1, 2], recovers the quantum state from complete observations of projection measurements of repeated preparations. For the state density matrix of $n$ qubits, the dimension is: $d=2^{n}$, and the number required for the complete measurement of the density matrix is: $d \times d=2^{n} \times 2^{n}=4^{n}$. Obviously, the number of measurements required for QST increases exponentially as the number of qubits increases [1], and the state reconstruction of QST is offline. For the state reconstruction of a high-qubits system, QST is more difficult to implement. In order to obtain the state information of a quantum system, people may introduce a detection system to associate with the quantum system, then measure the detection system directly. The information of an interested quantum state can be inferred by the measurement records of the detection system. If the interaction strength between the system and the detection system is rather weak, this kind of measurement is the weak measurement [3]. Quantum continuous weak measurement refers to continuous weak measurement of the quantum system, which can be used to realize the online estimation of quantum states. Compressed sensing (CS) provides a solution to the problem of reducing the number of measurements in quantum state estimation [4-6]. CS tells us that: if the rank of a state density matrix $r$ is much smaller than its dimension $d,(r<<d)$, then the state density matrix can be reconstructed with only a small amount of randomly sampled measurement records [7, 8]. Gross proved that one can reconstruct the state density matrix with only $O(r d \log d)$ measurement records using the Pauli measurement operators [9]. CS can be applied to the online estimation of quantum state and it improves the efficiency of calculation.

The feedback control of quantum system requires sys-

This work is supported by National Natural Science Foundation (NNSF) of China under Grant 61973290 and 61720106009 tem's state at any time. Therefore, online state estimation becomes the premise of quantum feedback control based on measurements $[10,11]$. With the development of the quantum control theory and quantum information technology, some researches have been achieved about the state transfer for the open quantum systems, such as, the state transfer of optimal control based on perturbation theory [12], the population transfer based on fast adiabatic process technology [13], the manipulations between eigenstates of the quantum system based on optimal measurements [14], and the state transfer based on Lyapunov stability theorem $[15,16]$. However, these researches were either for the open-loop control or closed-loop control based on the program. Therefore, how to use quantum continuous weak measurement to obtain state information, estimate quantum state online, and design an estimated state-based feedback quantum control system to realize the state transfer with high precision is a valuable and challenging research.

In this paper, we consider a two-level open quantum system with decoherence effect. The research task is to design a state feedback control law based on Lyapunov stability theorem to realize the state transfer from any initial state to any desired target state. In the research process, the indirect measurement with weak influence on the estimated state is used, and the measurement result containing the state information is obtained by continuously performing the weak measurement. The state estimation becomes an optimization problem after obtaining required measurement results. The compressed sensing theory is used for minimizing the number of the measurements when the density matrix has certain structure. Based on the estimated state, a state feedback controller for the state transfer is designed according to the Lyapunov stability theorem. Appropriate control parameters are determined in the simulation experiments, and three numerical simulation experiments are carried out for the states transfer from eigenstates to eigenstates, superposition states to superposition states, and superposition states to mixed states, respectively. The experimental results are also analyzed.

The structure of this paper is organized as follows: the procedure of the online estimated state-based feedback con- 
trol for an open quantum system is established in Section 2. In Section 3, the estimated state-based feedback control law is designed by means of the Lyapunov stability theorem. In Section 4, numerical simulation experiments are implemented in MATLAB environment, also the experimental results are analyzed. The full paper is summarized in Section 5.

\section{Online State Estimation for Feedback Control of Open Quantum System}

The structure of the online estimated state-based feedback control for an open quantum system is shown in Fig. 1, in which the controlled system is a two-level open quantum system with the decoherence effect, the part in dotted line is a complete online estimation process of quantum states, and the controller is a state feedback controller based on Lyapunov stability theorem. In this paper, the online estimated state of the quantum system is used to design the controller as feedback state.

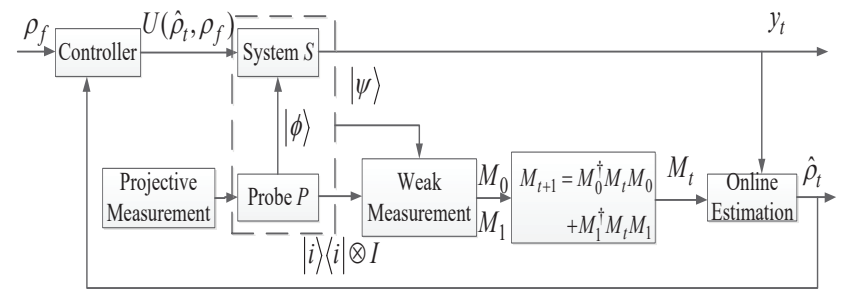

Fig. 1: Structure of the state transfer of the quantum system with online estimated state.

First, the dynamic equation of the controlled system in Fig. 1 can be written as:

$$
\begin{aligned}
& \rho(t+d t)-\rho(t)=-\frac{i}{\hbar}[H(t), \rho(t)] d t \\
& +\left[L \rho(t) L^{\dagger}-\left(\frac{1}{2} L^{\dagger} L \rho(t)+\frac{1}{2} \rho(t) L^{\dagger} L\right)\right] d t \\
& \rho(0)=\rho_{0}
\end{aligned}
$$

where, $\rho(t)$ represents the state density matrix of quantum system; $\hbar$ is Planck constant and let $\hbar=1$ for simplicity; $H(t)=H_{0}+\sum_{j=1}^{2} U_{j}(t) H_{j}$ is the whole Hamiltonian, $H_{0}$ is the free Hamiltonian, $H_{j}$ is the control Hamiltonian, and $H_{1}=\sigma_{x}, H_{2}=\sigma_{y}$, in which $\sigma_{x}, \sigma_{y}, \sigma_{z}$ are Pauli operators, $U_{j}(t)$ is the external control value for the state transfer; $L$ is the lindblad operator; let $D[L, \rho]=L \rho(t) L^{\dagger}-1 / 2 *$ $\left(L^{\dagger} L \rho(t)+\rho(t) L^{\dagger} L\right)$, which represents the deterministic decoherence effect brought by the measurement process and manifests as the drift term of the lindblad form.

In the online estimation of quantum state, a detection system is introduced to associate with the controlled system and the projection measurement is performed on the detection system. Then, the measurement operators acting on the controlled system can be derived based on the measurement results [17]. The measurement values that contain the state information of controlled system are obtained by means of measurement operators. Finally, the quantum state can be reconstructed by solving an optimization problem consisting of measurement values, measurement operators and system state. When performing continuous weak measurements on a one-qubit system, the measurement operator group contains only $M_{0}(\Delta t)$ and $M_{1}(\Delta t)$ operators according to the complete operator condition: $(M)^{2}+\left(M_{\perp}\right)^{2}=I$. By selecting the appropriate operator $L$, the corresponding weak measurement operators $M_{0}(\Delta t)$ and $M_{0}(\Delta t)$ can be constructed respectively:

$$
\begin{aligned}
& M_{0}(\Delta t)=I-\left(L^{\dagger} L / 2+i H(t)\right) \Delta t \\
& M_{1}(\Delta t)=L \cdot \sqrt{\Delta t}
\end{aligned}
$$

where, $I$ is a second-order identity matrix.

Combining the formula (2) and the system dynamics equations in formula (1), and let $t=\Delta t \cdot k$, the evolution equation in discrete form of open quantum system can be written as:

$$
\rho(k+1)=M_{0} \rho(k) M_{0}^{\dagger}+M_{1} \rho(k) M_{1}^{\dagger}
$$

where, $k=1,2, \ldots, N, N$ is the total number of system measurements and estimations in the simulation experiment.

The weak measurement operators are also constantly changing over time in the process of continuously weak measurement. According to the relationship between the measurement operator and the system state, the evolution equation in discrete form of the weak measurement operator can be written as:

$$
M(k+1)=M_{0}^{\dagger} M(k) M_{0}+M_{1}^{\dagger} M(k) M_{1}
$$

By using the measurement operators to make continuously weak measurement on the quantum system, the corresponding measurement values can be obtained. In theory, the measurement value can be obtained by calculating the inner product value of the density matrix $\rho$ and the measurement operator $M$, namely: $y=\operatorname{tr}\left(M^{\dagger} \rho\right)$. Therefore, the problem of reconstruction density matrix $\rho$ can be transformed into the following optimization problem: $\hat{\rho}=\arg \min \|\rho\|_{*}$ s.t. $\mathbf{y}=\mathbf{A v e c}(\rho)$, in which, $\|\rho\|_{*}$ is the kernel norm of $\rho, \hat{\rho}$ is the density matrix to be estimated, $\operatorname{vec}(\cdot)$ vectorizes a matrix to a column vector. The sampling matrix $\mathbf{A}$ is the matrix with all the sampled measurement operators $M(k): \mathbf{A}(k)=$ $(\operatorname{vec}(M(k)) \cdots \operatorname{vec}(M(2)) \operatorname{vec}(M(1)))^{\dagger}, k=$ $1,2, \ldots, m$. When the sampling time $k$ is greater than $m$, $\mathbf{A}(k)$ remains unchanged. We can obtain a measurement record sequence according to the measurement value $y(k)$ at each sampling time as $[y(1), \ldots, y(k-1), y(k)]=$ $\left[\operatorname{tr}\left(M(k)^{\dagger} \rho(k)\right), \ldots, \operatorname{tr}\left(M(2)^{\dagger} \rho(k)\right), \operatorname{tr}\left(M(1)^{\dagger} \rho(k)\right)\right]$. When the sampling time $k$ is greater than $m$, the number of the measurement record remains $m$.

When the quantum state is nearly pure, a nuclear norm ter$m$ can be added to the cost function. When consider a general case, the quantum state reconstructions problem can be written as an optimization function with quantum constraints on the density matrix as

$$
\begin{aligned}
& \hat{\rho}=\arg \min \|\mathbf{A} \cdot \operatorname{vec}(\rho)-\mathbf{y}\|_{2} \\
& \text { s.t. } \hat{\rho}=\rho, \rho \geq 0, \operatorname{tr}(\rho)=1
\end{aligned}
$$

For the online state estimation of the one-qubit quantum system in this paper, the system state in discrete form is $\rho(k)$. 
The measurement operator at any moment is $M(k)$, which is a $2 \times 2$ matrix, and we can obtain the sampling matrix $\mathbf{A}=\operatorname{vec}\left((M(k))^{\dagger}\right)$ with $4 \times 1$ dimension. The measurement value in the simulation experiment is: $\mathbf{y}=(y(k))$. After that, the LS optimization algorithm of CVX in MATLAB is used to solve the optimization problem in formula (5) to obtain the online estimated state $\hat{\rho}(k)$ of the quantum system.

\section{Design of State Feedback Controller Based on Online State Estimation}

The basic idea of using a quantum control method based on Lyapunov stability theorem is to select a Lyapunov $V(x)$ and make it satisfy $\dot{V}(x) \leq 0$. The key point is to select a suitable Lyapunov function [18]. Based on the real-time online estimated state of quantum system, the Lyapunov function based on state distance is selected as [19]:

$$
V=\frac{1}{2} \operatorname{tr}\left(\left(\hat{\rho}(t)-\rho_{f}\right)^{2}\right)
$$

where, $\hat{\rho}(t)$ is real-time estimated state of the quantum system, and $\rho_{f}$ is the desired target state.

The first derivative of the time obtained by the Lyapunov function $V$ is obtained:

$$
\begin{aligned}
& \dot{V}=\operatorname{tr}\left(\dot{\hat{\rho}}(t)\left(\hat{\rho}(t)-\rho_{f}\right)\right) \\
& =\sum_{j=1}^{2} U_{j}(t) \cdot \operatorname{tr}\left(\left(i\left[H_{j}, \hat{\rho}(t)\right]\right)\left(\hat{\rho}(t)-\rho_{f}\right)\right) \\
& +\operatorname{tr}\left(\left(D[L, \hat{\rho}(t)]-i\left[H_{0}, \hat{\rho}(t)\right]\right)\left(\hat{\rho}(t)-\rho_{f}\right)\right) \\
& =U_{1}(t) \cdot T_{1}+U_{2}(t) \cdot T_{2}+C
\end{aligned}
$$

where, $T_{j}=\operatorname{tr}\left(\left(i\left[H_{j}, \hat{\rho}(t)\right]\right)\left(\hat{\rho}(t)-\rho_{f}\right)\right), j=1,2$, is a real function on the online estimated state of the system; $C=\operatorname{tr}\left(\left(D[L, \hat{\rho}(t)]-i\left[H_{0}, \hat{\rho}(t)\right]\right)\left(\hat{\rho}(t)-\rho_{f}\right)\right)$, is the drift ter$\mathrm{m}$, and its sign is undeterminable; $U_{1}$ and $U_{2}$ are the control laws to be determined.

The main idea of designing the control laws in this paper is to offset the influence of the drift term $C$ by applying one of the control effects, and design another control action to make $\dot{V}(x) \leq 0$ hold. There is a fractional expression in the control laws: when $T_{1}$ and $T_{2}$ are both zero, $\dot{V}=C$, the mathematical calculation causes singularity due to the uncertainty of the value $C$. In this way, the negative semi-definite condition of $\dot{V}(x) \leq 0$ cannot always be established, and the method of designing the control laws based on Lyapunov stability theorem will no longer be applicable. Therefore, this paper introduces a threshold variable $\theta$ in the design of the control laws. By judging the size relationship between $T_{j}$ and $\theta$, it is divided into three cases, and the corresponding control action is designed to offset the drift term $C$ while satisfying $\dot{V}<0$ as much as possible, so that the value of the Lyapunov function $V$ is continuously reduced, and the desired control precision can be achieved. The specific design process of the control laws is as follows:

1) In equation (7), when the real function $\left|T_{1}\right|>\theta$, the design control law $U_{1}(t)=-C / T_{1}$ is used to cancel the drift term, and the design control law $U_{2}(t)=-g_{2} \cdot T_{2}$ is used to make $\dot{V}=-g_{2} \cdot T_{2}^{2} \leq 0$ hold. At this point the control laws can be written as:

$$
U(t)=\left[\begin{array}{l}
U_{1}(t) \\
U_{2}(t)
\end{array}\right]=\left[\begin{array}{l}
-C / T_{1} \\
-g_{2} \cdot T_{2}
\end{array}\right]
$$

where, $g_{2}$ is a positive adjustable control parameter.

2) When the real function $\left|T_{1}\right|<\theta,\left|T_{2}\right|>\theta$, the design control law $U_{2}(t)=-C / T_{2}$ is used to cancel the drift term, and the design control law $U_{1}(t)=-g_{1} \cdot T_{1}$ is used to make $\dot{V}=-g_{1} \cdot T_{1}^{2} \leq 0$ hold. At this point the control laws can be written as:

$$
U(t)=\left[\begin{array}{l}
U_{1}(t) \\
U_{2}(t)
\end{array}\right]=\left[\begin{array}{l}
-g_{1} \cdot T_{1} \\
-C / T_{2}
\end{array}\right]
$$

where, $g_{1}$ is a positive adjustable control parameter.

3) When $\left|T_{1}\right|<\theta$ and $\left|T_{2}\right|<\theta$, the value of the Lyapunov function $V$ is calculated to determine the degree of approximation of the system state to the desired target state. If the transfer error $\varepsilon_{0}$ is reached, the control target is considered to be implemented, and the controlled system has been transferred from the initial state to the target state, otherwise the values of the control parameters $g_{1}$ and $g_{2}$ are reselected.

The flow chart of the control laws based on the Lyapunov stability theorem is shown in Fig. 2. The execution condition of the dotted arrow needs to satisfy the following two cases: (1) when $\left|T_{1}\right|<\theta,\left|T_{2}\right|<\theta$ is established at the same time, and the transfer error is not within the allowable range of the control precision $\varepsilon_{0} ;(2)$ when the system evolves according to the control law designed under the conditions 1) and 2), the control target cannot be achieved. When the above two conditions occur, the values of the control parameters $g_{1}$ and $g_{2}$ need to be re-selected.

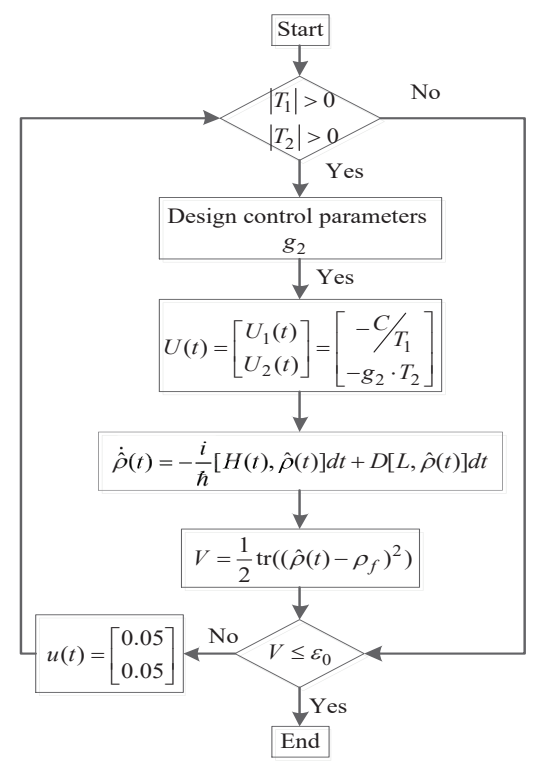

Fig. 2: Flow chart of designed control law.

\section{Simulation Experiments of State Transfer and Result Analysis}

In this section, the simulation experiments of state transfer are carried out in the MATLAB environment. The three state transfer are respectively from the eigenstate to the eigenstate, from the superposition state to the superposition state, and from the superposition state to mixed state, then the experimental results are analyzed, respectively.

In the online estimation process of the open quantum system based on continuous weak measurement, a 1/2 spin 
particle ensemble $\rho$ is considered as the object of the online estimation, which is under $z$ direction with a constant magnetic field $B_{z}$ and $x$ direction control magnetic field $B_{x}=A \cos \phi$. In Schrodinger picture, the initial state of the spin is $\rho_{0}$, and $\rho(k)$ represents the state at time of $k$. It is assumed that the damage caused by the measurement to the system is weak, that is, the weak measurement intensity tends to zero. Applying a continuous weak measure to the system $S$, the initial observation operator is $M(0)$. The experimental sampling period is $\Delta T=0.01$, and the threshold variable is $\theta=5 \times 10^{-4}$. The lindblad operator is $L=\sigma_{z}=\operatorname{diag}([1,-1])$ and the initial measurement operator is $M(0)=\sigma_{z}=\operatorname{diag}([1,-1])$. The expected state transition error of quantum system is taken as $\varepsilon_{0}=10^{-3}$ in the experiment.

\subsection{State transfer from eigenstate to eigenstate}

In this section, we mainly study the state transfer from the initial state $\rho_{0}=\operatorname{diag}([0,1])$ to the target state $\rho_{f}=$ $\operatorname{diag}([1,0])$ of the quantum system. Fig. 3 is the experimental results of the state transfer from the eigenstate to the eigenstate, where, Fig. 3(a) shows the state transfer trajectory on the Bloch ball, the red solid line corresponds to the transfer trajectory of system state in real time, the blue dashed line corresponds to the online estimated state, the red o represents the initial state $\rho_{0}$ of the system, the green $o$ represents the system target state $\rho_{f}$, and the blue $*$ represents the initial state of the real-time estimated state $\hat{\rho}_{0}$, Fig. 3(b) shows the variation of each element of the state density matrix of a one-bit system, Fig. 3 (c) shows the control laws curve, and Fig. 3(d) shows the variation curve of Lyapunov function, which represents the change of system transfer error $\varepsilon$. In this experiment, the parameters in the control law are respectively taken as $g 1=2, g 2=2.5$, the interaction intensity of continuous weak measurement is $\xi=0.2$, and the evolution time is $N=150$.

It can be seen from the simulation result curve of Fig. 3 that after 120 evolutions, the transfer error between the system state and the target state reaches $\varepsilon=2.6 \times 10^{-3}$, and the designed quantum Lyapunov control law based on online state estimation quickly realizes the state transfer from eigenstate to eigenstate.

In order to further verify the effectiveness of using the online estimation state as feedback state based on continuous weak measurement and compressed sensing theory, we We calculate the system fidelity to indicate the performance of online estimation of the quantum state. The formula for defining fidelity is defined as: $f(k)=$ $\operatorname{Tr} \sqrt{\hat{\rho}(k)^{\frac{1}{2}} \rho(k) \hat{\rho}(k)^{\frac{1}{2}}}$, where, $\rho(k)$ is the true density matrix of the system at time $k$ and $\hat{\rho}(k)$ is the corresponding online estimated density matrix. Fig. 4 is the fidelity curve of online estimation of quantum state.

From Fig. 4, we can see that the fidelity of the estimated state reaches above $99.4 \%$. It means that online estimation of quantum state can be achieved by the continuous weak measurement and compressed sensing theory. Moreover, it's effective and achievable to use the online estimated state for designing the state feedback control law.

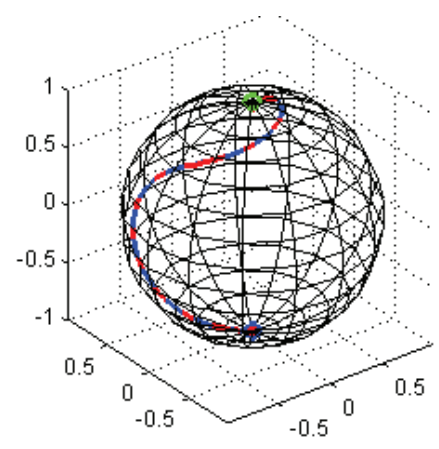

(a) State transfer trajectory on the Bloch ball

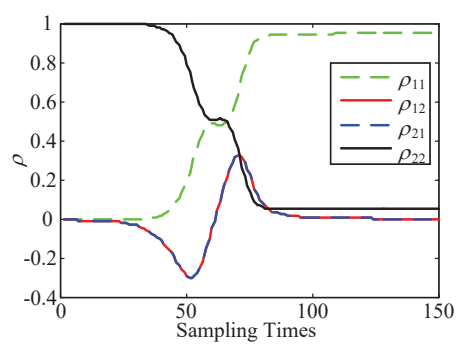

(b) Variation of each element of the state density matrix

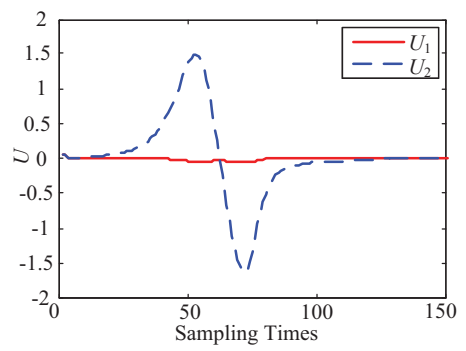

(c) Control laws curve

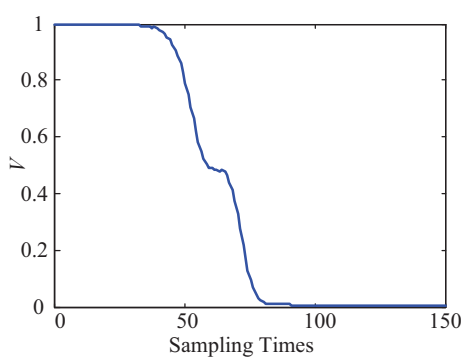

(d) Variation curve of Lyapunov function

Fig. 3: Experiment results of the eigenstate transfer.

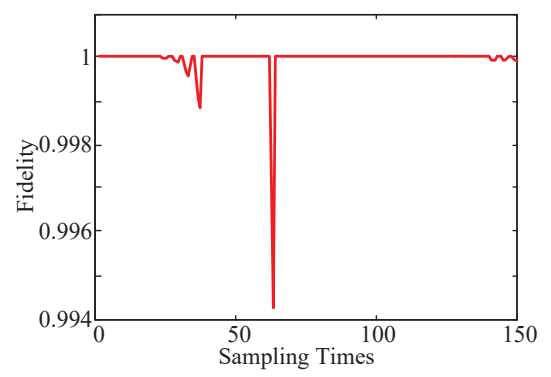

Fig. 4: Fidelity curve of online estimation of quantum states. 


\subsection{State transfer from superposition state to superpo- sition state}

In this experiment, the initial state of quantum system is selected as superposition state $\rho_{0}=[15 / 16 \sqrt{15} / 16 ; \sqrt{15} / 161 / 16]$ and the target state is also superposition state $\rho_{f}=[3 / 8-\sqrt{15} / 8 ;-\sqrt{15} / 85 / 8]$. Fig. 5 is the experiment result of the state transfer from superposition state to superposition state.

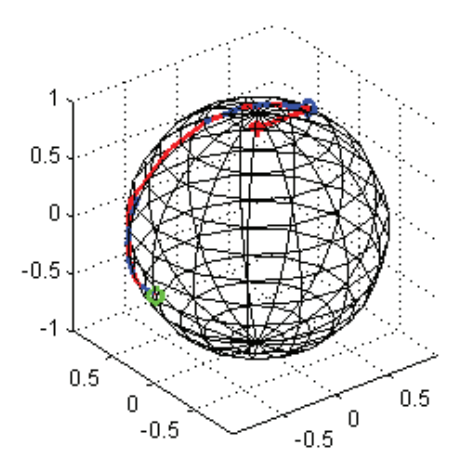

(a) State transfer trajectory on the Bloch ball

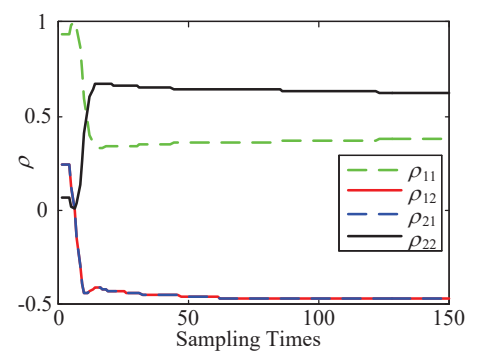

(b) Variation of each element of the state density matrix

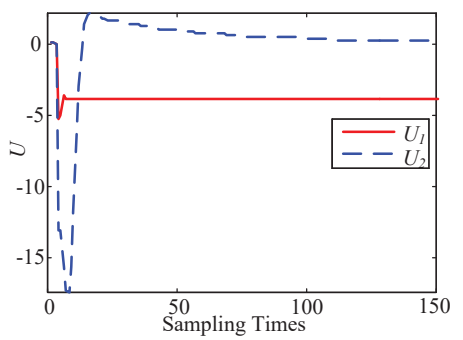

(c) Control laws curve

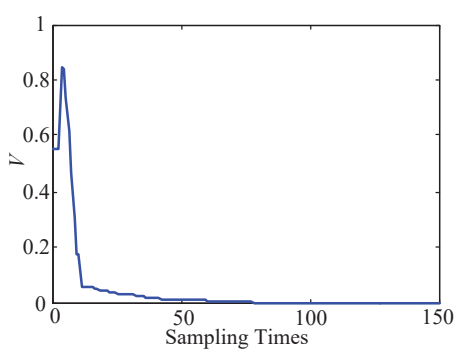

(d) Variation curve of Lyapunov function

Fig. 5: Experiment results of the superposition state transfer.

Fig. 5(a) shows the state transfer trajectory on the Bloch ball, Fig. 5(b) shows the variation of each element of the state density matrix of a single-bit system, Fig. 5(c) shows the control law curve, and Fig. 5(d) shows the variation curve of Lyapunov function. In this experiment, the parameters in the control law are respectively taken as $g 1=2, g 2=18$, the interaction intensity of continuous weak measurement is $\xi=0.1$, and the evolution time is $N=150$.

It can be seen from the simulation experimemtal results of Fig. 5 that after 140 evolutions, the transfer error between the system state and the target state reaches $\varepsilon=4 \times 10^{-4}$. Comparing Fig. 3(c) with Fig. 5(c), it can be seen that the state transfer from the superposition state to the superposition state requires a larger amount of control than the state transfer from the eigenstate to the eigenstate. When the system state moves to the target state, the control is still needed to maintain the state.

\subsection{State transfer from superposition state to mixed s- tate}

In this section, we mainly study the state transition from the initial superposition state $\rho_{0}=[3 / 8-\sqrt{15} / 8 ;-\sqrt{15} / 85 / 8]$ to the mixed state $\rho_{f}=[3 / 51 / 5 ; 1 / 52 / 5]$. Fig. 6 is the experiment result of the state transfer from superposition state to mixed state, where, Fig. 6(a) shows the state transfer trajectory on the Bloch ball, Fig. 6(b) shows the variation of each element of the state density matrix of a one-bit system, Fig. 6(c) shows the control laws curve, and Fig. 6(d) shows the variation curve of Lyapunov function. In this experimen$\mathrm{t}$, the parameters in the control law are respectively taken as $g 1=2, g 2=16$, the interaction intensity of continuous weak measurement is $\xi=0.45$, and the evolution time is $N=250$.

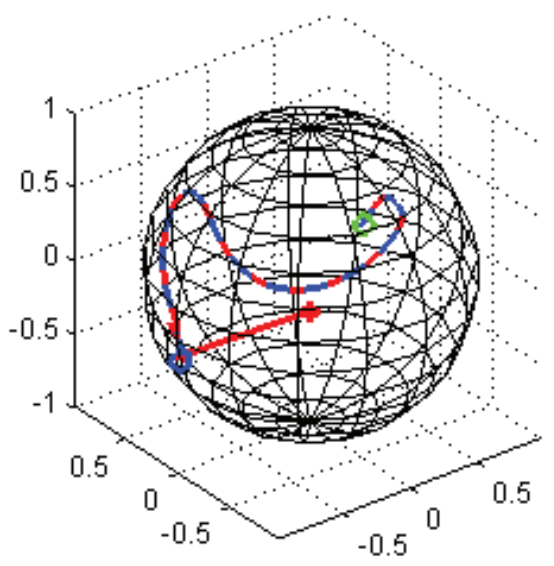

(a) State transfer trajectory on the Bloch ball

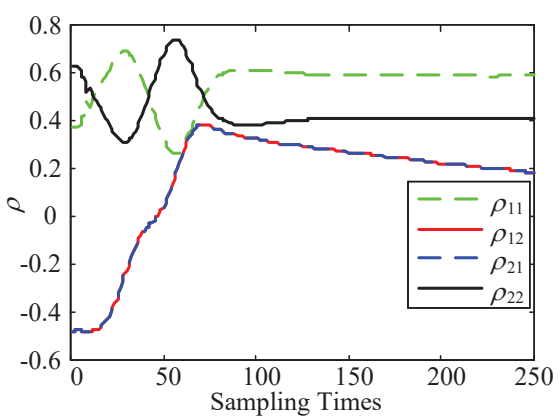

(b) Variation of each element of the state density matrix 


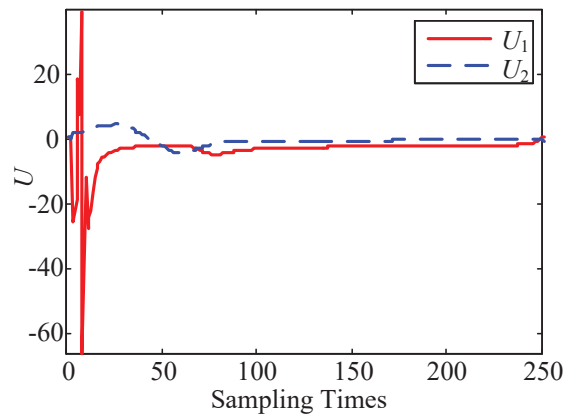

(c) Control laws curve

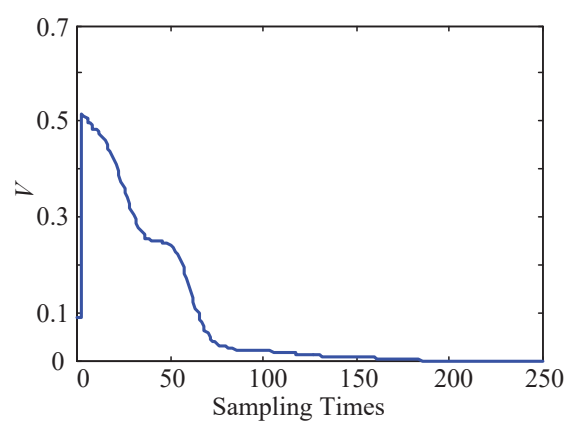

(d) Variation curve of Lyapunov function

Fig. 6: Experiment results of state transfer from superposition state to mixed state.

It can be seen from the simulation results of Fig. 6 that when the target state of the system is mixed state, the quantum Lyapunov control laws can effectively change the evolution trajectory of the system state and realize the state transfer from superposition state to mixed state. After 230 evolutions, the transfer error between the system state and the target state reaches $\varepsilon=5 \times 10^{-4}$.

The experimental results show that for the online estimation state of the system obtained by quantum continuous weak measurement and compressed sensing theory, the control law designed based on Lyapunov stability theory can realize the state transfer from different initial states to the target states of open quantum system. Compared with the state transition between the eigenstates, the control required for the state transfer from the superposition state to the superposition state and the mixed state is stronger, and the time required is longer.

\section{Conclusion}

The control laws proposed in this paper was based on the online state estimation and the Lyapunov stability theorem to realize the state feedback control of the open quantum state. Numerical simulation experiments were done to verify the effectiveness of using the designed control laws for the system state transfer. Moreover, the state feedback control of the open quantum system based on online quantum state estimated proposed in this paper can be extended to the state transfer control for the multiple-qubit systems.

\section{References}

[1] D. D'Alessandro, On quantum state observability and measurement, Journal of Physics A: Mathematical and General, vol. 36, no. 37, pp. 9721-9735, 2003.
[2] G. M. D’Ariano, Paris M. G. A, and Sacchi M. F, Quantum tomographic methods, Lecture Notes in Physics, vol. 649, pp. $7-58,2004$.

[3] O. Oreshkov, T. A. Brun, Weak Measurements Are Universal., Physical Review Letters, vol. 95, no. 11, pp. 110409, 2005.

[4] E. J. Cands, J. Romberg and T. Tao, Robust uncertainty principles: Exact signal reconstruction from highly incomplete frequency information, IEEE Transactions on information theory, vol. 52, no. 2, pp. 489-509, 2006.

[5] K. Zheng, K. Li, and S. Cong, A reconstruction algorithm for compressive quantum tomography using various measurement sets, Scientific Reports, vol. 6, pp. 38497, 2016.

[6] K. Li, H. Zhang, S. Kuang, F. Meng, and S. Cong, An Improved Robust ADMM Algorithm For Quantum State Tomography, Quantum Information Processing, vol. 15, no. 6, pp. 2343-2358, 2016.

[7] J. Zhang, K. Li, S. Cong, H. Wang, Efficient reconstruction of density matrices for high dimensional quantum state tomography, Signal Processing, vol. 139, pp. 136-142, 2017.

[8] K. Li, J. Zhang, S. Cong, Fast reconstruction of high-qubitnumber quantum states via low-rate measurements, Physical Review A, vol. 96, no. 1, pp. 012334, 2017.

[9] D. Gross, Recovering low-rank matrices from few coefficients in any basis, IEEE Transactions on Information Theory, vol. 57, no. 3, pp. 1548-1566, 2011.

[10] C. J. Bardeen, V. V. Yakovlev, K. R. Wilson, S. D. Carpenter, P. M. Weber, and W. S. Warren, Feedback quantum control of molecular electronic population transfer Chemical Physics Letters, vol. 280, no. 1, pp. 151-158, 1997.

[11] Q. Sun, I. Pelczer, G. Riviello, R. B. Wu, and H. Rabitz, Experimental observation of saddle points over the quantum control landscape of a two-spin system, Phys. Rev. A, vol. 91, no. 4, pp. 043412, 2015.

[12] B. M. Escher, G. Bensky, J. Clausen, and G. Kurizki, Optimized control of quantum state-transfer from noisy to quiet qubits, Journal of Physics B: Atomic, Molecular and Optical Physics, vol. 44, no. 15, pp. 154015, 2011.

[13] W. Cui, Z. R. Xi, and Y. Pan, Controlled population transfer for quantum computing in Non-Markovian noise environmen$\mathrm{t}$, Proceeding of Joint 48th IEEE Conference on Decisionand Control and 28th Chinese Control Conference, pp. 2504-2509, 2009.

[14] J. Yang, S. Cong, Feng Shuang, and Herschel Rabitz, Manipulations between eigenstates of 2-level quantum system based on the optimal measurement, IEEE/CAA JOURNAL OF AUTOMATICA SINICA, vol. 3, no. 1, pp. 35-41, 2016.

[15] S. Kuang, and S. Cong, Lyapunov control methods of closed quantum systems, Automatica, vol. 44, no. 1, pp. 98-108, 2008.

[16] N. Ghaeminezhad, and S. Cong, Preparation of Hadamard Gate for Open Quantum System by Lyapunov Control Method, IEEE/CAA JOURNAL OF AUTOMATICA SINICA, vol. 5, no. 3, pp. 733-740, 2018.

[17] A. Silberfarb, P. Jessen, and I. H. Deutsch, Quantum state reconstruction via continuous measurement, Physical Review Letters, vol. 95, no. 3, pp. 030402, 2005.

[18] S. Cong, State Manipulation in Lyapunov-based Quantum System Control Methods, Control Theory and Applications, vol. 29, no. 3, pp. 273-281, 2012.

[19] S. Cong, L. Hu, F. Yang, and J. Liu, Characteristics Analysis and State Transfer for non-Markovian Open Quantum Systems, ACTA AUTOMATICA SINICA, vol. 39, no. 4, pp. 360-370, 2013. 